\title{
Resonant Rydberg-atom-microwave-field interactions in the ultrastrong-driving regime: Beyond the rotating-wave approximation
}

\author{
J. D. R. Tommey and S. D. Hogan \\ Department of Physics and Astronomy, University College London, Gower Street, London WC1E 6BT, United Kingdom
}

(Received 24 June 2019; published 25 November 2019)

\begin{abstract}
The coherent interaction of Rydberg atoms with microwave fields in the ultrastrong-driving regime, in which the Rabi frequency is of the same order of magnitude as the transition frequency, has been studied for states with principal quantum number $n=105$ in helium. Experiments were performed in pulsed supersonic beams, and the effects of the ultrastrong $1.280 \mathrm{GHz}$ microwave driving field, tuned to near resonance with the $1 s 105 s^{3} S_{1} \rightarrow 1 s 105 p^{3} P$ transition, were identified from Autler-Townes splittings of the $1 s 3 p^{3} P_{2} \rightarrow 1 s 105 s^{3} S_{1}$ transition by cw laser spectroscopy. The microwave field strength was calibrated in situ in the apparatus from Autler-Townes splittings measured in the weak-driving regime in which the rotating-wave approximation holds. The results of the experiments were compared to the energy-level structure of the atoms in the presence of the microwave field calculated using Floquet methods. From this comparison, the microwave-field strengths for which the rotating-wave approximation and the two-level approximation break down have been identified. The feasibility of implementing microwave traps for Rydberg atoms and molecules, which operate in the ultrastrong-driving regime, has been evaluated.
\end{abstract}

DOI: 10.1103/PhysRevA.100.053417

\section{INTRODUCTION}

Highly excited Rydberg states of atoms and molecules can possess exaggerated properties and interactions with external fields. This has seen them exploited as controllable model systems with which to study many strong-field phenomena in the laboratory, including, for example, tunnel ionization in strong electric [1-4] and nonresonant microwave fields [5-7], diamagnetic interactions in strong magnetic fields [8,9], and electric dipole interactions with other single particles including other Rydberg atoms [10,11], ground-state polar molecules [12-15], and photons [16,17]. Here we employ high Rydberg states in helium, with principal quantum number $n=105$, to investigate coherent atom-electromagnetic-field interactions in the ultrastrong-driving regime. This regime is characterized by Rabi oscillation frequencies $\Omega$ that are of the same order of magnitude as the atomic transition frequency $v_{0}$. In this situation, the rotating-wave approximation (RWA) [18], widely used to simplify theoretical treatments of the interaction of two-level quantum systems with resonant electromagnetic fields, breaks down. This ultrastrong-driving regime of atommicrowave-field interactions is distinct from the strong-field regimes investigated previously with atomic Rydberg systems; see, e.g., Ref. [19].

Strong $\left(\Omega \sim 0.1 v_{0}\right)$ and ultrastrong $\left(\Omega \sim v_{0}\right)[20]$ coherent interactions of atoms with electromagnetic fields, and the detailed evaluation of methods for calculating the effects of these interactions on the atomic energy-level structure, are of interest in a wide range of research areas. In quantum information processing and, most recently, matter-wave interferometry experiments with atoms in Rydberg states [21], resonant electromagnetic fields are used to prepare quantum states by Rabi flopping. The implementation of faster state preparation protocols requires the use of stronger fields even- tually approaching the regime in which the RWA must break down [22]. Rydberg atoms which possess large electric dipole transition moments, and a wide range of allowed electric dipole transition frequencies, make for excellent microwavefield sensors $[23,24]$. A detailed understanding of strong- and ultrastrong-field interactions is essential in determining the limits of the dynamic range of sensors of this kind [25]. The use of strong off-resonant microwave dressing fields has been investigated as an approach to minimizing, or canceling, the static electric dipole polarizabilities of low- and high-angularmomentum Rydberg states to decrease their sensitivity to stray static fields [26], e.g., in hybrid cavity quantum electrodynamics (QED) experiments [27-29]. Microwave traps operating in the strong-driving regime [30] are of interest in the preparation of cold samples of Rydberg atoms and molecules for applications in quantum information processing [31,32] and studies of chemical dynamics at low temperatures [32].

The remainder of this article is structured as follows: In Sec. II, the numerical Floquet methods used to calculate the Rydberg energy-level structure and spectral intensities up to the ultrastrong microwave driving regime are described. The techniques and apparatus used in the experiments are then presented in Sec. III. In Sec. IV A, the results of studies carried out in the weak-driving regime, to calibrate in situ the microwave-field strength through measurements of AutlerTownes splittings, are discussed. In Sec. IV B, the effects of ultrastrong driving are presented, and the regimes in which the RWA and two-level approximation are suitable for describing the atom-microwave-field interaction are identified. In Sec. V, the feasibility of using ultrastrong microwave-field interactions for trapping Rydberg atoms and molecules is discussed, before conclusions are drawn in Sec. VI. 


\section{FLOQUET CALCULATIONS}

The Hamiltonian describing the interaction of a Rydberg atom with an oscillating electric field, e.g., that associated with an electromagnetic field at microwave frequencies, can be expressed as

$$
H_{\mathrm{tot}}(t)=H_{0}+H_{\mathrm{osc}}(t),
$$

where $H_{0}$ is the zero-field Hamiltonian and $H_{\mathrm{osc}}(t)$ represents the interaction with the field. Because of the weak spin-orbit couplings in high- $n$ Rydberg states, which scale as $n^{-3}$ and give rise to energy-level splittings of $<100 \mathrm{kHz}$ for the triplet Rydberg states in helium with $n=105$ studied here [33], the Schrödinger equation associated with this Hamiltonian can be solved in an $\left|n, \ell, m_{\ell}\right\rangle$ basis $\left(\ell\right.$ and $m_{\ell}$ are the electron orbital angular momentum and azimuthal quantum numbers, respectively).

To treat the time-dependent perturbation associated with the interaction of the atom with the microwave field in Eq. (1), Floquet's theorem is invoked. This states that the eigenfunctions $\Psi_{\kappa}$ of a Hamiltonian $H(t)=H(t+T)$ which is periodic in time, where $T$ is the periodicity, are also time periodic, i.e., $[34,35]$

$$
\Psi_{\kappa}(t+T)=\Psi_{\kappa}(t)=e^{i \epsilon_{\kappa} t / \hbar} \Phi_{\kappa}(t) .
$$

Here, $\Phi_{\kappa}(t)$ is the Floquet mode, a time-dependent superposition of the field-free basis states. Each Floquet mode has an associated time-independent quasienergy $\epsilon_{\kappa}$, which is not uniquely defined but has values

$$
\epsilon_{\kappa}=\epsilon_{\kappa_{0}}+q \hbar \omega_{d},
$$

where $q$ is an integer, $\omega_{d}=2 \pi \nu_{\mathrm{d}}=2 \pi / T$, and $\hbar$ is the reduced Planck constant. The value of $q$ corresponds to the number of photons contributing to the mode. The Schrödinger equation associated with this time-periodic Hamiltonian can be rewritten in terms of the quasienergies and Floquet modes, i.e., $\mathcal{H}(t) \Phi_{\kappa}(t)=\epsilon_{\kappa} \Phi_{\kappa}(t)$, and can therefore be solved by calculating the eigenvalues of the matrix associated with the Floquet Hamiltonian operator $\mathcal{H}(t)=H(t)-i \hbar \partial_{t}$. In principle, $\mathcal{H}(t)$ is of infinite dimension; in practice, however, multiphoton interactions beyond a certain order do not influence the dynamics and the matrix may be truncated without loss of accuracy.

To transform from $H(t)$ to $\mathcal{H}(t)$, the photon number states are accounted for through the quantum number $q$, where $-\infty<q<\infty$, giving a total Hilbert space for the atom in the presence of the field spanned by basis states of the form $\left|n, \ell, m_{\ell}, q\right\rangle$. In this Floquet basis, the diagonal elements of the matrix $\mathcal{H}(t)$, for high Rydberg states in helium, can be expressed as

$$
\left\langle n, \ell, m_{\ell}, q\left|\mathcal{H}_{0}\right| n, \ell, m_{\ell}, q\right\rangle=-\frac{R_{\mathrm{He}} h c}{\left(n-\Delta_{n, \ell}\right)^{2}}+q h v_{\mathrm{d}},
$$

where $R_{\mathrm{He}}$ is the Rydberg constant corrected for the reduced mass of helium, and $h$ and $c$ are the Planck constant and the speed of light in vacuum, respectively. The quantum defects $\Delta_{n, \ell}$ of the triplet Rydberg states in helium with $n=105$ are listed in Table I.

For a microwave electric field linearly polarized in the $z$ dimension, $H_{\mathrm{osc}}(t)=z e F_{\mathrm{osc}} \cos \left(2 \pi v_{\mathrm{d}} t\right)$. Upon transformation
TABLE I. Quantum defects of the triplet Rydberg states in helium with $n=105$ [36].

\begin{tabular}{lc}
\hline \hline$\ell$ & $\Delta_{105 \ell}$ \\
\hline 0 & 0.296660 \\
1 & 0.068359 \\
2 & 0.002891 \\
3 & 0.000447 \\
4 & 0.000127 \\
5 & 0.000049 \\
6 & 0.000023 \\
\hline \hline
\end{tabular}

into spherical polar coordinates, the matrix elements associated with the time average of this operator can be separated into radial and angular parts and written in the Floquet basis as [35,37]

$$
\begin{aligned}
& \left\langle n^{\prime}, \ell^{\prime}, m_{\ell}^{\prime}, q^{\prime}\left|\mathcal{H}_{\mathrm{osc}}\right| n, \ell, m_{\ell}, q\right\rangle \\
& \quad=\delta_{q^{\prime}, q \pm 1} \frac{e F_{\mathrm{osc}}}{2}\left\langle n^{\prime}, \ell^{\prime}|r| n, \ell\right\rangle\left\langle\ell^{\prime}, m_{\ell}^{\prime}|\cos \theta| \ell, m_{\ell}\right\rangle,
\end{aligned}
$$

where $e$ is the electron charge. The radial integrals $\left\langle n^{\prime}, \ell^{\prime}|r| n, \ell\right\rangle$ in this expression are determined using the Numerov method [23,38]. The angular integrals $\left\langle\ell^{\prime}, m_{\ell}^{\prime}|\cos \theta| \ell, m_{\ell}\right\rangle$ are calculated analytically using standard angular momentum algebra [38,39]. The angular integrals associated with the $\cos \theta$ operator are only nonzero if $\Delta \ell=\ell^{\prime}-\ell= \pm 1$ and $\Delta m_{\ell}=m_{\ell}^{\prime}-m_{\ell}=0$. In Eq. (5), the Kronecker $\delta$ function, $\delta_{q^{\prime}, q \pm 1}$, restricts the couplings between Floquet states to those for which a single photon is exchanged with the microwave field, i.e., $\Delta q=q^{\prime}-q= \pm 1$. In general, to achieve convergence to within the experimental precision in the calculations of the energy-level structure of the triplet Rydberg states in helium with $n=105$ and a single value of $m_{\ell}$ studied here, a basis of states with $103 \leqslant n \leqslant 107, \quad 0 \leqslant \ell \leqslant 8, \quad$ and $-10 \leqslant q \leqslant+10$ was required. In the following, when only sublevels with $m_{\ell}=0$ are considered, the notation used to label the resulting basis states has been reduced to $|n \ell, q\rangle$.

After constructing the complete Hamiltonian matrix $\mathcal{H}(t)=\mathcal{H}_{0}+\mathcal{H}_{\mathrm{osc}}(t)$ in the Floquet basis, the energy-level structure of the dressed atom in the presence of the microwave field is calculated by determining its eigenvalues. The spectral intensities of electric dipole transitions from the $1 s 3 p^{3} P_{2}$ level to the resulting eigenstates were calculated from the corresponding eigenvectors as described in Ref. [37]. Although the complete basis of field-free states described above is required to accurately calculate the atomic energy-level structure in the presence of a microwave field, a general picture of the effect of the field can be obtained by considering a simpler two-level system composed only of the triplet $|105 s\rangle$ and $|105 p\rangle$ states. The transition between these two field-free states occurs at a frequency of $1.304 \mathrm{GHz}$. The corresponding energy-level structure in the presence of a microwave field at this frequency, i.e., when $v_{\mathrm{d}}=v_{0}=$ $1.304 \mathrm{GHz}$, is presented in Fig. 1. For weak microwave driving, i.e., when $F_{\text {osc }}<100 \mathrm{mV} / \mathrm{cm}$, the quasienergy levels of this atomic two-level system depend linearly on the field strength. Because $v_{\mathrm{d}}=v_{0}$, the $|105 s, q\rangle$ and $|105 p, q-1\rangle$ 


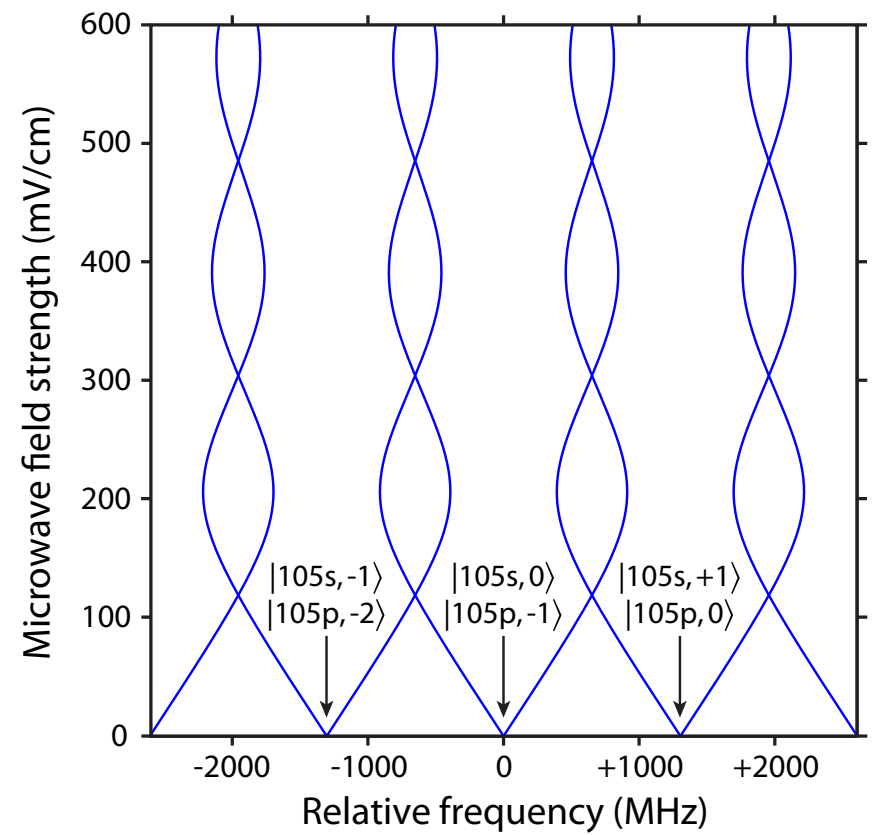

FIG. 1. Floquet energy levels of a two-level system composed of the triplet $|105 s\rangle$ and $|105 p\rangle$ states in helium in the presence of a resonant $1.304 \mathrm{GHz}$ microwave field. The frequency on the horizontal axis is displayed with respect to that of the field-free $|105 s, 0\rangle$ state.

basis states are degenerate when $F_{\mathrm{osc}}=0 \mathrm{~V} / \mathrm{cm}$. In the presence of the field, these pairs of basis states are coupled. The resulting eigenstates that emerge and split as $F_{\text {osc }}$ is increased are the symmetric and antisymmetric combinations of these two basis states. For $F_{\text {osc }}>100 \mathrm{mV} / \mathrm{cm}$, the splittings between these pairs of superposition states exceed $v_{0}$, and avoided crossings occur. These arise because of the second-order interactions between the corresponding states that are a consequence of sequential $\Delta \ell= \pm 1, \Delta q= \pm 1$ couplings between Floquet states as described by Eq. (5). These avoided crossings are a signature of the ultrastrongdriving regime of the atom-microwave-field interaction in which the splittings of the Floquet quasienergy levels is of the order of the field-free atomic transition frequency. In this regime, the dependence of the energy-level structure of the atom on the value of $F_{\text {osc }}$ reduces, and the intervals between the eigenstates tend towards a constant value corresponding to $v_{\mathrm{d}}$.

In addition to their large electric dipole transition moments, atoms in high Rydberg states can possess large static electric dipole moments and static electric dipole polarizabilities. Therefore, uncontrolled stray static (dc) electric fields cannot be neglected in experiments. Under the conditions of the experiments performed here, stray electric fields can be compensated in the $z$ dimension, but residual perpendicular fields remain [33]. To account for the effects of these and other weak electric fields applied in the $z$ direction, the Hamiltonian in Eq. (1) is extended such that

$$
H(t)=H_{0}+H_{\mathrm{osc}}(t)+H_{\mathrm{S}}
$$

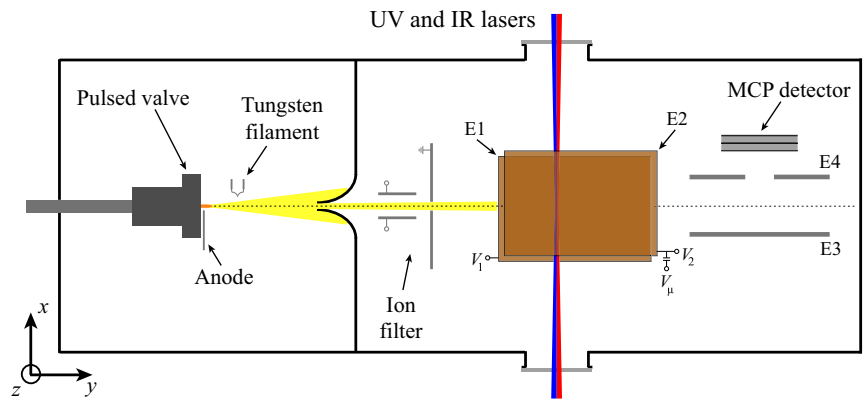

FIG. 2. Schematic diagram of the experimental apparatus.

where $H_{\mathrm{S}}=e \vec{F} \cdot \vec{r}$ contains contributions from both parallel and perpendicular static electric field components, i.e., $\vec{F}=\left(F_{x}, 0, F_{z}\right)$. Following the same procedure employed in constructing the Hamiltonian matrix associated with $H_{\mathrm{osc}}(t)$, $H_{\mathrm{S}}$ may be converted to $\mathcal{H}_{\mathrm{S}}$ in the Floquet basis, such that

$$
\begin{aligned}
& \left\langle n^{\prime}, \ell^{\prime}, m_{\ell}^{\prime}, q^{\prime}\left|\mathcal{H}_{\mathrm{S}}\right| n, \ell, m_{\ell}, q\right\rangle \\
& =e \delta_{q, q^{\prime}}\left\langle n^{\prime}, \ell^{\prime}|r| n, \ell\right\rangle\left[F_{x}\left\langle\ell^{\prime}, m_{\ell}^{\prime}|\sin \theta \cos \phi| \ell, m_{\ell}\right\rangle+\cdots\right. \\
& \left.\quad+F_{z}\left\langle\ell^{\prime}, m_{\ell}^{\prime}|\cos \theta| \ell, m_{\ell}\right\rangle\right] .
\end{aligned}
$$

The corresponding matrix elements are only nonzero when they couple Floquet states of the same sideband order, i.e., when $\Delta q=0$. The angular integrals in Eq. (7) result in selection rules for the couplings of $\Delta \ell= \pm 1$, and $\Delta m_{\ell}=0, \pm 1$ for parallel and perpendicular fields, respectively.

\section{EXPERIMENTAL APPARATUS}

A schematic diagram of the experimental apparatus is shown in Fig. 2. A pulsed supersonic beam of helium atoms in the metastable $1 s 2 s^{3} S_{1}$ level was generated in an electric discharge at the exit of a pulsed valve [40]. The beam, with a mean longitudinal speed of $2000 \mathrm{~m} / \mathrm{s}$, passed through a 2-mm-diameter skimmer before entering a region in which an electric field was generated to deflect and filter ions created in the discharge. The atoms then entered between two parallel $70 \times 105 \mathrm{~mm}$ copper plates, E1 and E2, which were separated by $13 \mathrm{~mm}$ in the $z$ dimension. In this region, copropagating UV and IR laser beams intersected the atomic beam. The UV laser was stabilized to $\lambda_{\mathrm{uv}}=388.9751 \mathrm{~nm}$ to drive the $1 s 2 s^{3} S_{1} \rightarrow 1 s 3 p^{3} P_{2}$ transition. The IR laser was tuned in the range from 785.1669 to $785.1710 \mathrm{~nm}$ to drive $1 s 3 p^{3} P_{2} \rightarrow 1 s 105 s^{3} S_{1} / 1 s 105 d^{3} D$ transitions, and probe the effects of microwave fields on the energy-level structure of the atoms [41]. On entering the vacuum chamber, the UV and IR laser beams had powers of 4 and $300 \mathrm{~mW}$, respectively, and were focused to full-width-at-half-maximum beam waists of $\sim 100 \mu \mathrm{m}$. The microwave fields were generated between E1 and E2 by capacitively coupling the output of a microwave source directly to electrode E2. IR laser photoexcitation spectra recorded in the experiment allowed the Autler-Townes splitting [42] of the $1 s 3 p^{3} P_{2} \rightarrow 1 s 105 s^{3} S_{1}$ transition in the presence of a $1.280 \mathrm{GHz}$ microwave field, near resonant with the $1 s 105 s^{3} S_{1} \rightarrow 1 s 105 p^{3} P(|105 s\rangle \rightarrow$ $|105 p\rangle$ ) transitions at $1.304 \mathrm{GHz}$, to be probed. This particular microwave frequency was chosen to exploit a resonance in the 
microwave circuit between the signal generator and electrodes in the apparatus. Following Rydberg-state photoexcitation, the excited atoms traveled out from between E1 and E2 and into the detection region of the apparatus between E3 and E4. To minimize losses by multiphoton microwave ionization during this $65-\mu \mathrm{s}$-long period of free flight, the microwave source was operated in a pulsed mode. It was switched on $10 \mu$ s before the velocity class of atoms selected for detection reached the photoexcitation lasers, and switched off $20 \mu \mathrm{s}$ later. Unless stated otherwise, for all experiments reported here, static offset potentials of $V_{1}=-V_{2}=12.5 \mathrm{mV}$ were applied to E1 and E2 to compensate stray electric fields in the $z$ direction between these electrodes. When the atoms reached the detection region, a pulsed voltage of $+295 \mathrm{~V}$ was applied to electrode E3 while E4 was maintained at $0 \mathrm{~V}$. The electric field generated by this voltage pulse ionized the excited Rydberg atoms and accelerated the resulting ions through an aperture in E4 to a microchannel plate (MCP) detector.

\section{RESULTS}

To study the effects of ultrastrong microwave driving on the triplet Rydberg states in helium with $n=105$, a first set of experiments were performed in the regime in which $\Omega \ll v_{0}$ to carry out in situ calibration of the microwave field strength in the laser photoexcitation region of the apparatus. These studies were then extended to the ultrastrong-driving regime in which $\Omega \gtrsim v_{0}$ and over a larger spectral range to investigate the breakdown of the two-level approximation, and the breakdown of the rotating-wave approximation in this system. The results of all the experiments that were performed were compared to the results of the calculations that were carried out using the Floquet methods described in Sec. II.

\section{A. Microwave-field strength calibration}

Rydberg states are routinely used as microscopic antenna for low-frequency electromagnetic fields [24]. This sensitivity was exploited in the present experiments to perform in situ calibration of the $v_{\mathrm{d}}=1.280 \mathrm{GHz}$ microwave-field strength in the laser photoexcitation region of the apparatus by measuring the Autler-Townes splitting of the $1 s 3 p^{3} P_{2} \rightarrow$ $1 s 105 s^{3} S_{1}$ transition upon weak microwave driving, i.e., when $\Omega \simeq 40 \mathrm{MHz} \ll v_{0}=1.304 \mathrm{GHz}$. In this regime, the atom-microwave-field interaction can be accurately treated within the RWA and the relationship between the AutlerTownes splitting and microwave-field strength is therefore given by $\Omega=\sqrt{\Omega_{0}^{2}+\Delta \nu^{2}}$ [43], where $\Omega$ is the splitting between the Autler-Townes components, $\Delta v=v_{\mathrm{d}}-v_{0}$ is the detuning of the microwave field from resonance, and $\Omega_{0}=$ $|\langle 105 p|e \hat{z}| 105 s\rangle| F_{\text {osc }} / h$ is the product of the transition dipole moment and the microwave-field strength. Measurements of this Autler-Townes splitting for a fixed value of $F_{\text {osc }}$ are presented in Fig. 3 (continuous curves). The relative frequency on the horizontal axis in this figure is displayed with respect to the zero-field $1 s 3 p^{3} P_{2} \rightarrow 1 s 105 s^{3} S_{1}$ transition frequency.

In recording the data in Fig. 3, a resonance in the microwave circuitry was exploited to maximize the strength of the microwave field that could be generated in the laser

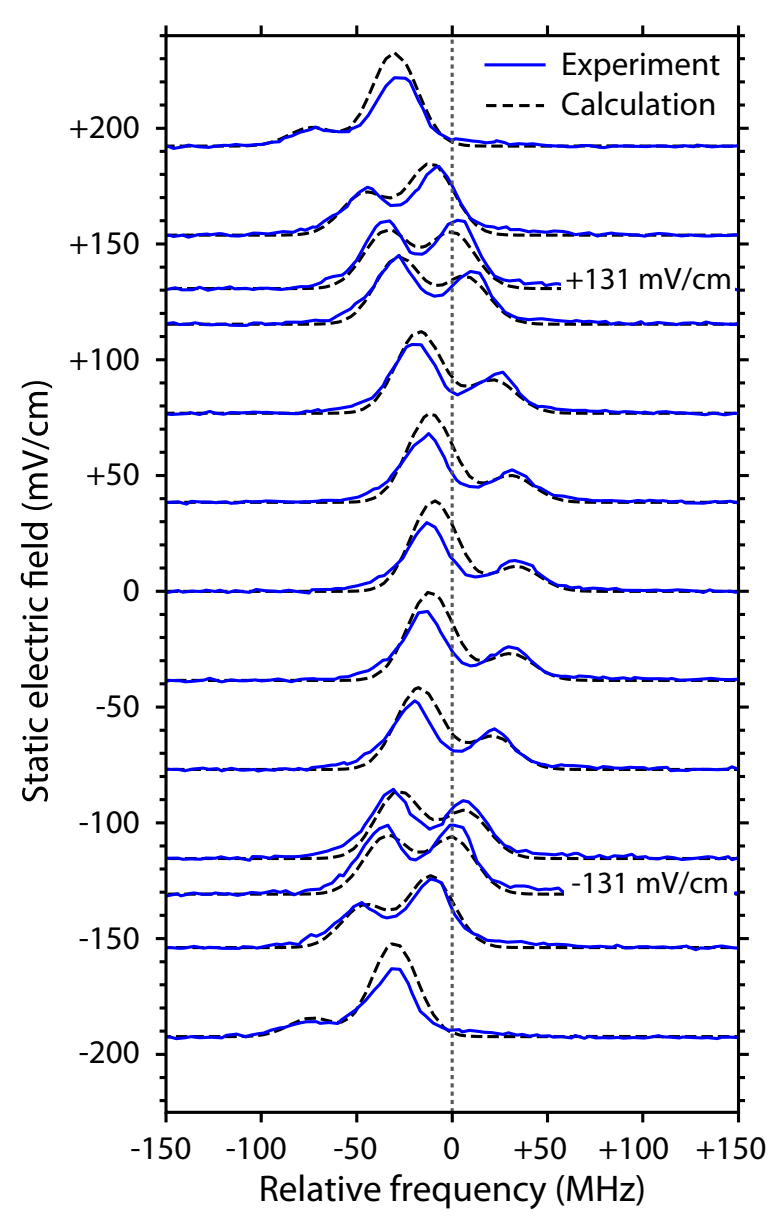

FIG. 3. Measured (continuous curves) and calculated (dashed curves) spectra of the $1 s 3 p^{3} P_{2} \rightarrow 1 s 105 s^{3} S_{1}$ transition in helium in the presence of a weak $v_{\mathrm{d}}=1.280 \mathrm{GHz}$ microwave field generated for an output power of $-26.5 \mathrm{dBm}(2.2 \mu \mathrm{W})$ from the source. The spectra were recorded in a range of dc electric fields as indicated by the vertical offset in each case.

photoexcitation region. This resonance at $1.280 \mathrm{GHz}$ occurred $24 \mathrm{MHz}$ below $v_{0}$. Because of this detuning, the laser spectrum of the transition to the Autler-Townes split |105s level in the zero dc electric field, seen in the middle of Fig. 3, exhibits an asymmetric intensity distribution. The relative amplitude of the two Autler-Townes components depends on the value of $\Delta v$. Since $\Delta v<0$, the lower-frequency component exhibits greater $|105 s\rangle$ character and, therefore, a higher spectral intensity. To study the effect of the microwave detuning from the $|105 s\rangle \rightarrow|105 p\rangle$ atomic transition frequency on the laser spectra, measurements were performed in a range of $\mathrm{dc}$ electric fields. As the dc offset field was adjusted, the $|105 s\rangle \rightarrow|105 p\rangle$ transition underwent a quadratic Stark shift toward lower frequency. This caused the transition to move through resonance with the fixed-frequency microwave field. The resonance condition, for which $\Delta v=0$, occurred for $F_{z}= \pm 131 \mathrm{mV} / \mathrm{cm}$. This can be seen from the corresponding spectra of the Autler-Townes splitting in Fig. 3. As $\left|F_{z}\right|$ approaches $131 \mathrm{mV} / \mathrm{cm}$ in these data, the spectral intensity distributions of the Autler-Townes components becomes increasingly symmetric. The two spectral features have equal intensity when $\left|F_{z}\right|=131 \mathrm{mV} / \mathrm{cm}$, while for larger values of 
(a)

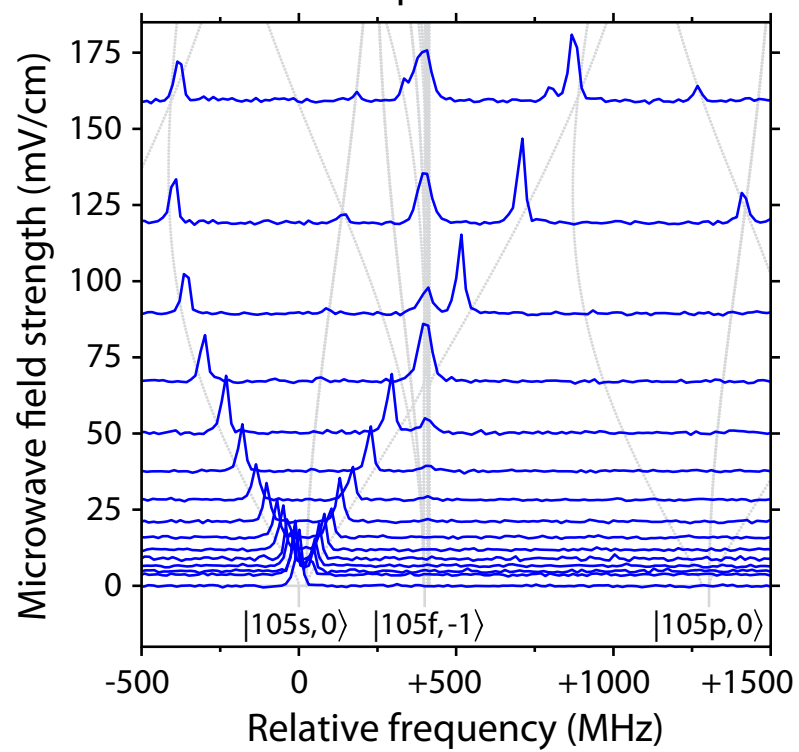

(b)

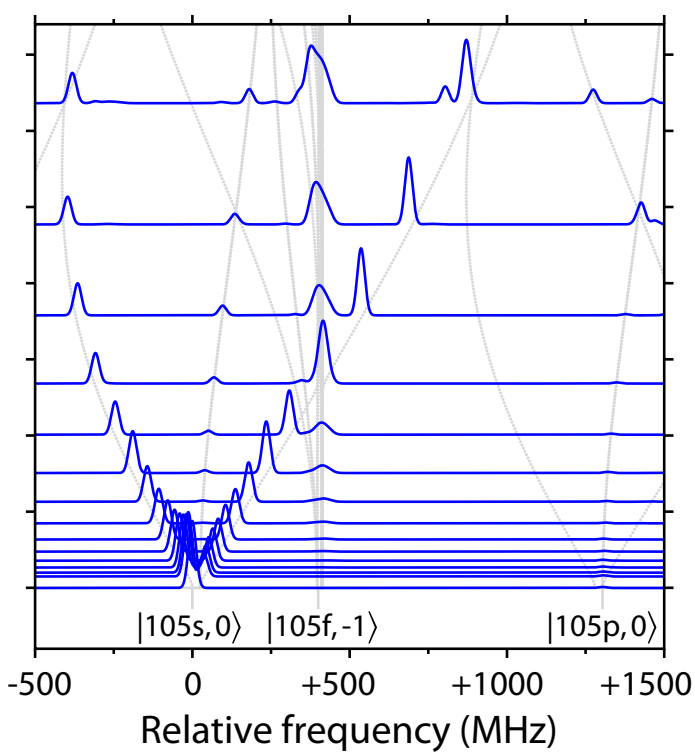

FIG. 4. (a) Experimentally recorded and (b) calculated spectra of transitions from the $1 s 3 p^{3} P_{2}$ level in helium to Rydberg states with $|n s, 0\rangle$ or $|n d, 0\rangle$ character in the presence of a $v_{\mathrm{d}}=1.280 \mathrm{GHz}$ microwave field. The microwave-field strength for which each spectrum was recorded is indicated by the vertical offset in each case. The thin gray curves in both panels represent the energy-level structure calculated using a reduced set of basis states (see text for details).

$\left|F_{z}\right|$, the detuning $\Delta v$ is positive and the asymmetric spectral intensities of the Autler-Townes components invert. Under these conditions, the higher-frequency components exhibit greater $|105 s\rangle$ character and hence intensity in the spectra.

The experimental data in Fig. 3 have been compared to the results of numerical calculations (dashed curves). These calculations were performed using the methods described in Sec. II with a small basis of states for which $n=105,0 \leqslant \ell \leqslant$ $6,-\ell \leqslant m_{\ell} \leqslant+\ell$, and $-5 \leqslant q \leqslant+5$, and in which the only free parameter was a single scaling factor used to convert the square root of the microwave power output from the source to $F_{\text {osc }}$. By performing a global fit to all of the experimental data in Fig. 3, the field strength at the position of the atoms in the experiment was determined to be $3.68(17) \mathrm{mV} / \mathrm{cm}$. This field corresponds to a microwave intensity in the laser photoexcitation region of the apparatus of $1.80(17) \times 10^{-8} \mathrm{~W} \mathrm{~cm}^{-2}$ and was generated when the output power of the microwave source was $-26.5 \mathrm{dBm}(2.2 \mu \mathrm{W})$.

\section{B. Ultrastrong microwave driving}

Laser spectra recorded for microwave source output powers from -26.5 to $+9 \mathrm{dBm}$, and hence $F_{\text {osc }}=3.7$ to $159 \mathrm{mV} / \mathrm{cm}$, are displayed in Fig. 4(a). For the weakest microwave fields employed when recording the data in this figure, the asymmetric intensity distributions of the AutlerTownes components of the transition to the $|105 s\rangle$ level are clearly seen close to zero-frequency detuning. This asymmetry is also visible in the results of calculations performed for the corresponding microwave-field strengths in Fig. 4(b). The calculated spectra in Fig. 4(b) were obtained using the full basis of $|n \ell, q\rangle$ states listed in Sec. II and a contribution from an uncanceled perpendicular stray electric field of $F_{x}=2$ $\mathrm{mV} / \mathrm{cm}$ (see Sec. II). However, to aid in the interpretation of the spectra, the energy-level structure indicted by the thin gray curves was calculated using a reduced basis for which $n=105,0 \leqslant \ell \leqslant 6,-\ell \leqslant m_{\ell} \leqslant+\ell$, and $-5 \leqslant q \leqslant+5$. The results of these calculations are also overlaid on the experimental data in Fig. 4(a). As the value of $F_{\text {osc }}$ was increased in the experiments toward $50 \mathrm{mV} / \mathrm{cm}$, the separation between the two Autler-Townes components, which corresponds to the Rabi frequency $\Omega$, increases approximately linearly. Close to $50 \mathrm{mV} / \mathrm{cm}$, an additional feature, indicated by the broad vertical gray bar, at a relative frequency of $+400 \mathrm{MHz}$ becomes visible. This spectral feature is detuned by $-v_{\mathrm{d}}$ from the interval between the $1 s 3 p^{3} P_{2}$ level and the $1 s 105 f^{3} F$ levels. In the spectrum, this feature therefore represents a non-resonance-enhanced two-color, infrared-plus-microwave, two-photon transition from the $1 s 3 p^{3} P_{2}$ level to the $1 s 105 f^{3} F$ levels.

For values of $F_{\text {osc }}$ greater than $50 \mathrm{mV} / \mathrm{cm}$ in Fig. 4(a), the shift in the Autler-Townes component of the transition to the $|105 s\rangle$ level, which is at negative relative frequency, deviates from a linear dependence on the microwave-field strength. In these higher microwave fields, other spectral features also appear, e.g., additional peaks can be seen close to $+1250 \mathrm{MHz}$. These additional features correspond to transitions to the $1 s 105 d^{3} D$ levels which undergo an ac Stark shift in the microwave field. In general, there is very good quantitative agreement between the experimental data and the results of the calculations over the full range of microwavefield strengths studied in Fig. 4. The only slight discrepancies between the results of the experiments and the results of the calculations become apparent in the relative intensities of the spectral features associated with the highest values of $F_{\text {osc }}$. To identify the origins of these discrepancies, test calculations were performed with larger computational bases and including effects of the diamagnetic interaction of the 


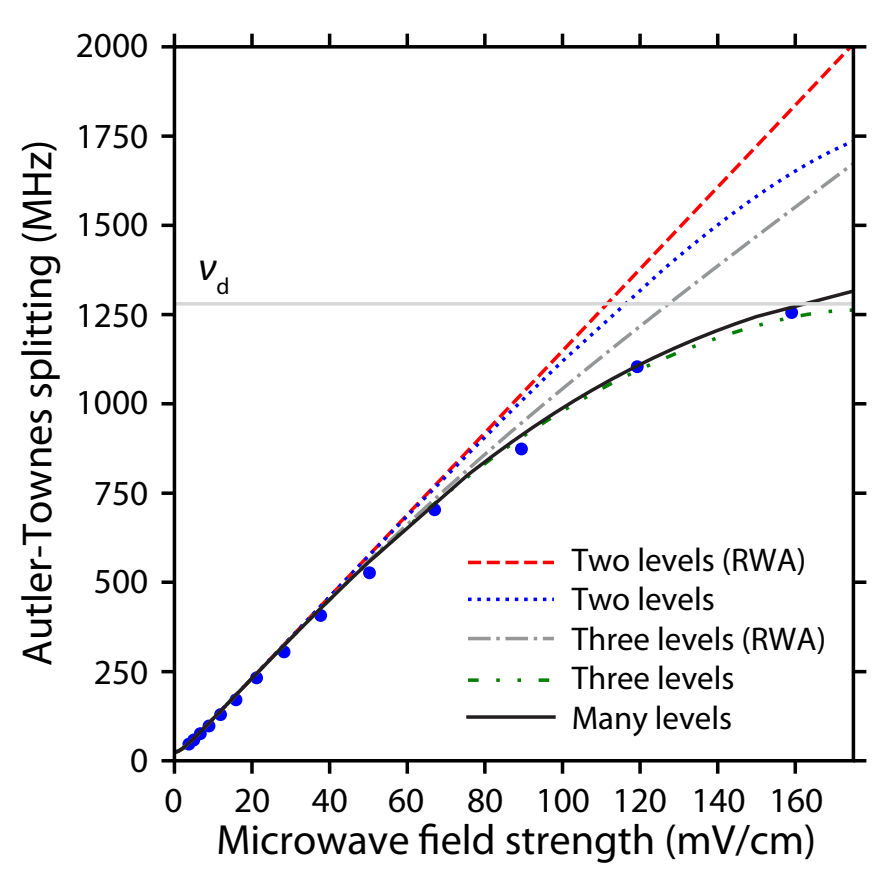

FIG. 5. Dependence of the Autler-Townes splitting of the $1 s 3 p^{3} P_{2} \rightarrow 1 s 105 s{ }^{3} S_{1}$ transition on the strength of the $1.280 \mathrm{GHz}$ microwave field. The splittings determined from the experimental data in Fig. 4 are indicated by the blue points. Note that the error bars associated with these measured splittings are smaller than the points.

atoms with the microwave field. These did not yield improved agreement between the experimental and calculated data. It was therefore concluded that a reduction in the spectral purity of the microwave field in the apparatus at high operating powers was the most likely cause of the observed discrepancies.

The Autler-Townes splittings in the spectra in Fig. 4 contain information that can be used to evaluate the ranges of validity of simplified models of the atom-microwave-field interaction. The Autler-Townes model is applicable for a twolevel system within the RWA. However, as can be concluded from the studies presented in Fig. 4, levels other than the $|105 s\rangle$ and $|105 p\rangle$ levels must contribute to the measured spectra particularly for the higher values of $F_{\text {osc }}$ considered. An assessment of the applicability of the RWA and fewlevel approximations in simplifying the description of the Rydberg-atom-microwave-field interaction can be made from an analysis of the Autler-Townes splitting of the transition to the $|105 s\rangle$ state. To allow a quantitative comparison to be made, the experimentally measured $|105 s\rangle \rightarrow|105 p\rangle$ Rabi frequencies, determined from the Autler-Townes splittings of the $1 s 3 p^{3} P_{2} \rightarrow 1 s 105 s^{3} S_{1}$ transition in the spectra in Fig. 4(a) are displayed in Fig. 5 as the blue points, and compared to theoretical models for either two- or three-level systems with and without the RWA.

Up to the strong-driving regime in Fig. 5, i.e., for $F_{\text {osc }} \lesssim$ $50 \mathrm{mV} / \mathrm{cm}\left(\Omega \lesssim v_{\mathrm{d}} / 2\right)$, the approximately linear increase in the observed Autler-Townes splitting as the microwavefield strength was increased suggests that the RWA and two-level approximation are both valid. In the Floquet calculations, the RWA was implemented by coupling only cer- tain Floquet states. In this situation, nonenergy conserving interactions, which are only important on short timescales, were neglected. Thus, only the $|105 s, q\rangle \rightarrow|105 p, q-1\rangle$ and $|105 p, q\rangle \rightarrow|105 s, q+1\rangle$ couplings were considered. The Autler-Townes splitting calculated within the RWA and two-level approximation for an electric dipole transition moment of $|\langle 105 p|e \hat{z}| 105 s\rangle|=8970 e a_{\mathrm{He}}$ associated with the field-free $|105 s\rangle \rightarrow|105 p\rangle$ transition $\left(a_{\mathrm{He}}\right.$ is the Bohr radius corrected for the reduced mass of helium) is indicated by the dashed red curve in Fig. 5 and is in good agreement with the experimental data for $F_{\text {osc }} \lesssim 50 \mathrm{mV} / \mathrm{cm}$. The blue dotted curve in the figure shows the splitting expected for the same two-level system, but with all non-RWA couplings allowed and for which $-10 \leqslant q \leqslant+10$. The dash-dotted and dash-double-dotted curves correspond to calculations for three-level systems composed of the $|105 s\rangle,|105 p\rangle$, and $|105 d\rangle$ states with three Floquet modes, i.e., the $|105 s, 0\rangle$, $|105 p,-1\rangle$, and $|105 d,-2\rangle$ modes (RWA), and $-10 \leqslant q \leqslant$ 10 (non-RWA), respectively. The Autler-Townes splittings and hence the Rabi frequencies obtained from these threelevel calculations are both in good quantitative agreement with the experimental data up to $F_{\text {osc }} \simeq 80 \mathrm{mV} / \mathrm{cm}$. For field strengths beyond this, the RWA breaks down, predicting a linear increase in $\Omega$, while the experimental data begin to saturate towards $\Omega=v_{\mathrm{d}}$. The non-RWA calculations for the $|105 s\rangle,|105 p\rangle$, and $|105 d\rangle$ three-level system do, however, reflect the observed splitting up to the final measured data point at $F_{\text {osc }} \simeq 150 \mathrm{mV} / \mathrm{cm}$, and only deviate from the full many-level calculation (continuous curves) at these highest microwave field strengths.

A notable difference between the results of the RWA and non-RWA calculations in Fig. 5 relates to the saturation of the Autler-Townes splitting at high values of $F_{\text {osc }}$. As seen in Fig. 1, in a complete non-RWA calculation at high powers, the $q= \pm 1$ sidebands associated with each field-free level undergo avoided crossings. In the ultrastrong-driving regime, these avoided crossings lead to an energy-level structure dominated by integer multiples of the drive frequency. The RWA decouples the levels that undergo these avoided crossings and the resulting energy-level structure arises, e.g., in the particular case considered here from the coupling of the $|105 s, 0\rangle$ level to the $|105 p,-1\rangle$ level, which itself is coupled to the $|105 d,-2\rangle$, etc., in a ladder configuration. The neglected couplings in the RWA mean that instead of undergoing avoided crossings, the energy levels continue to diverge as they cross levels associated with other sideband orders. The saturation of the Autler-Townes peaks seen in the experimental data in Figs. 4 and 5 is therefore a signature of the breakdown of the RWA.

From the data in Fig. 5, it can be concluded that the two-level approximation is valid up to the regime in which $\Omega \simeq 0.5 v_{\mathrm{d}}$. In this strong-driving regime, the role of the Bloch-Siegert shift of the atomic resonance frequency on the measured Rabi frequency may therefore be investigated $[44,45]$. The Bloch-Siegert shift arises from the higher-order corrections to the transition frequency of a pure two-level system that occurs under strong resonant driving. To identify corrections associated with the Bloch-Siegert shift that may be observable in the experimental data in Fig. 4, calculations were performed to determine the non-RWA contributions 


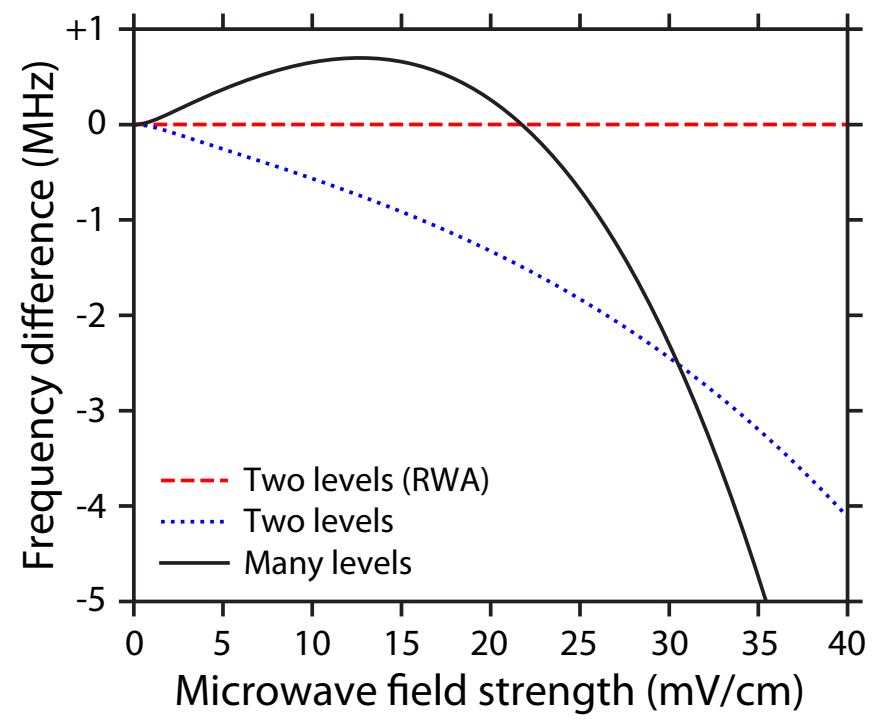

FIG. 6. Calculated deviation of the Autler-Townes splitting in weak microwave fields from that obtained under the RWA. Results obtained for a two-level (many-level) system with $-10 \leqslant q \leqslant 10$ are indicted by the dotted curve (continuous curve).

to the Autler-Townes splitting in the two-level system. The results of these calculations are shown in Fig. 6. In this figure, the energy-level splitting calculated under the RWA is subtracted from the results of each calculation. Hence, for all microwave-field strengths, the change in the Autler-Townes splitting in the pure two-level system under the RWA is zero (dashed line). The remaining difference in the Autler-Townes splitting, obtained for a pure two-level system but including interactions beyond the RWA, is indicated by the dotted curve. From this data, it is seen that if the system under study was a pure two-level system, the Autler-Townes splitting, and hence the Rabi frequency, would be smaller than predicted under the RWA when the Bloch-Siegert shift becomes significant. However, as can be seen from the results of the calculations performed by determining the eigenvalues of the complete multilevel Floquet Hamiltonian with $-10 \leqslant q \leqslant+10$, and indicated by the continuous curve in Fig. 6, the AutlerTownes splitting in this setting increases more rapidly than that predicted under the RWA in weak fields up to $15 \mathrm{mV} / \mathrm{cm}$. Consequently, the contributions from the third level in the system studied here dominate effects arising from the BlochSiegert shift. This is a similar situation to that encountered in studies of ultrastrongly driven quantum wells [46]. In the case of interest here, we conclude that under the conditions in which the measurements were performed, the two-level approximation breaks down before the RWA does, masking the effect of the Bloch-Siegert shift.

\section{APPLICATION TO MICROWAVE TRAPPING OF RYDBERG ATOMS AND MOLECULES}

The ultrastrong near-resonant Rydberg-atom-microwavefield interactions reported here are relevant to the realization of microwave traps for cold Rydberg atoms and molecules. Such devices are expected to find applications in hybrid cavity QED experiments with atoms in circular Rydberg
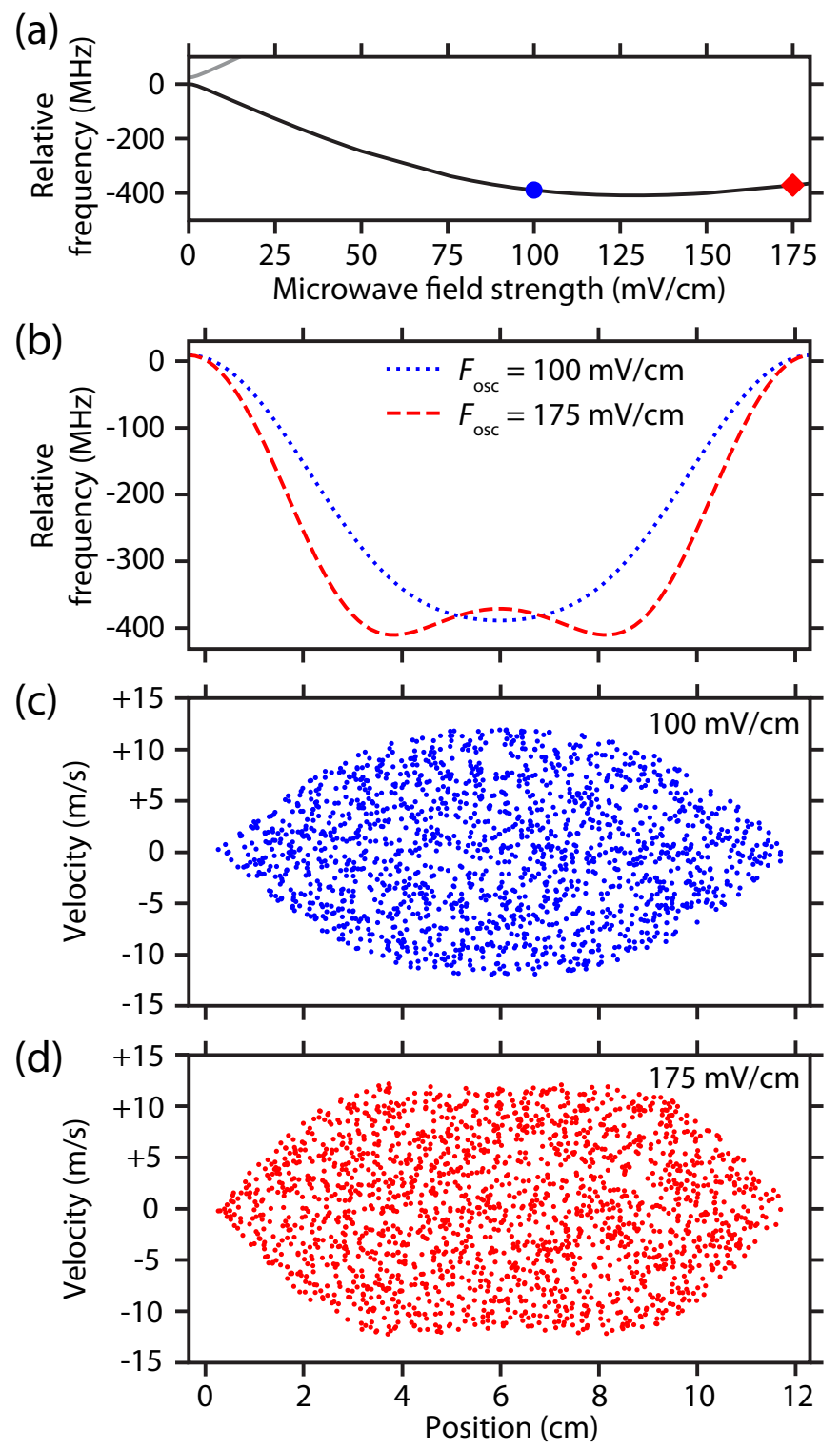

FIG. 7. (a) Energy shift of the triplet $|105 s\rangle$ level in helium in the presence of a $1.280 \mathrm{GHz}$ microwave field. (b) Corresponding potential-energy distribution for this atom in the HFS Autler-Townes component near the $0.10 \mathrm{~V} / \mathrm{cm}$ (dashed curve) or $0.175 \mathrm{~V} / \mathrm{cm}$ (dotted curve) intensity maxima of a single antinode of this field. (c),(d) One-dimensional (1D) phase-space acceptance of a trap based on the potentials in (b) as indicated.

states and superconducting microwave circuits $[28,29,47]$. This approach to trapping exploits the force $\vec{f}=-\nabla W$ on an atom or molecule in a spatially inhomogeneous microwave field, where $W$ is the potential energy of the atom in the field. Forces of this kind lead to the acceleration of samples with positive- (negative)-energy shifts in the presence of the microwave field, toward regions of low- (high)-field strength. The corresponding states are therefore referred to as low-fieldseeking (LFS) and high-field-seeking (HFS) states. As can be inferred from the data in Fig. 4, the LFS or HFS character of a particular field-free energy level, e.g., the $|105 s\rangle$ level in the presence of a strong near-resonant microwave field, can 
be selected through the appropriate choice of the detuning of the microwave field from resonance. Therefore, this approach to controlling the motion of Rydberg atoms and molecules is particularly versatile and applicable to a wide range of states, including nondegenerate states which are purely HFS in static electric fields, i.e., low- $\ell$ Rydberg states in nonhydrogenic atoms or molecules, or circular states.

Microwave decelerators have been implemented for ground-state polar molecules [30]. In these devices, the field distribution in a cylindrical microwave resonator was employed, and tuned below resonance, to prepare and decelerate HFS ammonia molecules. To determine the phase-space acceptance of a microwave trap for helium atoms in the high- $n$ Rydberg states considered here, numerical particle trajectory simulations in a trap, based on the parameters of the experiments reported in Sec. IV A, were carried out. As in Sec. IV A, the detuning of $v_{\mathrm{d}}-v_{0}=-24 \mathrm{MHz}$, meant that atoms prepared in the $|105 s\rangle$ level were HFS. The adiabatic evolution of the resulting dressed $|105 s\rangle$ level in the microwave field is shown in Fig. 7(a). The detuning $\Delta v$ plays an important role in negating nonadiabatic population transfer when atoms traverse regions of low-field strength. In a resonant field, the two Autler-Townes components would become degenerate when the microwave-field strength approaches zero, and population transfer to the LFS component could occur. In this situation, atoms that undergo this population transfer would be ejected from a trap. Control over the microwave frequency therefore provides (i) the ability to choose which free-field state is addressed, and (ii) provides a method to protect atoms in this selected state from nonadiabatic trap loss. Figure 7(b) shows the potential energy of a helium atom initially prepared in the field-free $|105 s\rangle$ level over the full length of a microwave trap, i.e., over a distance of half the wavelength of a standing wave microwave field, for the two field strengths of 100 and $175 \mathrm{mV} / \mathrm{cm}$, indicated by the points in Fig. 7(a). The blue dotted curve in Fig. 7(b) corresponds to a maximum field strength of $100 \mathrm{mV} / \mathrm{cm}$; in this situation, the trap has a single minimum located at its center where the field amplitude is greatest. The dashed red curve corresponds to a maximum field strength of $175 \mathrm{mV} / \mathrm{cm}$, for which the state has gone through an avoided crossing and has become LFS [see Fig. 7(a)]. This second case results in a trap in which two minima form, one on either side of the center. This nonmonotonic evolution of the atomic energy-level structure in the microwave field therefore allows tuneable trap geometries to be realized by adjusting only the amplitude of the microwave field. The phase-space acceptances in these two trapping configurations are shown in Figs. 7(c) and 7(d), respectively. The maximum speed of the atoms that can be trapped in these traps is $\sim 10 \mathrm{~m} / \mathrm{s}$. This corresponds to a translational temperature of $E_{\text {kin }} / k_{\mathrm{B}} \simeq$ $25 \mathrm{mK}$. The trap depths are directly related to the energy shifts of the Rydberg state in the microwave field as this represents the maximum kinetic energy that a trapped atom can have without escaping from the trap. Additional calculations (not shown) indicate that the corresponding situation for the $|50 s\rangle$ and $|50 p\rangle$ levels would result in trap depths of $\sim 300 \mathrm{mK}$ for $v_{\mathrm{d}}=12.150 \mathrm{GHz}$ and $F_{\mathrm{osc}} \simeq 5 \mathrm{~V} / \mathrm{cm}$. These trap depths are well suited to match the phase-space distributions of samples decelerated or transported in transmission-line decelerators $[48,49]$.

\section{CONCLUSIONS}

In conclusion, we have observed coherent Rydberg-atommicrowave-field interactions in the ultrastrong-driving regime for which $\Omega \sim v_{0}$. The experimental data are in excellent quantitative agreement with the results of numerical calculations of the energy-level structure, and spectral intensities, of the atom dressed by the microwave field which were performed using Floquet methods. These calculations have been compared to simpler models within the two-level and rotating-wave approximations and demonstrate that for the Rydberg states used in the experiments, these approximations are valid provided $\Omega \lesssim 0.5 \nu_{0}$. The results presented are of importance for applications of Rydberg atoms in the detection and precise calibration of microwave fields. The thorough understanding of the energy-level structure of high Rydberg states in the ultrastrong-driving regime obtained in this work has allowed an evaluation of microwave trapping schemes for the confinement of cold Rydberg atoms and molecules. The trap depths that are expected to be achieved in such devices, based on the parameters of the experiments reported here, are appropriate for applications in hybrid quantum information processing and the preparation of cold samples of Rydberg atoms and molecules.

\section{ACKNOWLEDGMENTS}

This work was supported by the EPSRC Centre for Doctoral Training in Delivering Quantum Technologies at University College London, and by the European Research Council (ERC) under the European Union's Horizon 2020 research and innovation program (Grant No. 683341).
[1] T. W. Ducas, M. G. Littman, R. R. Freeman, and D. Kleppner, Stark Ionization of High-Lying States of Sodium, Phys. Rev. Lett. 35, 366 (1975).

[2] W. van de Water, D. R. Mariani, and P. M. Koch, Ionization of highly excited helium atoms in an electric field, Phys. Rev. A 30, 2399 (1984).

[3] J. Grimmel, M. Stecker, M. Kaiser, F. Karlewski, L. TorralboCampo, A. Günther, and J. Fortágh, Ionization spectra of highly
Stark shifted rubidium Rydberg states, Phys. Rev. A 96, 013427 (2017).

[4] K. Gawlas and S. D. Hogan, Rydberg-state ionization dynamics and tunnel ionization rates in strong electric fields, Phys. Rev. A 99, 013421 (2019).

[5] M. W. Noel, L. Ko, and T. F. Gallagher, Microwave Ionization of an Atomic Electron Wave Packet, Phys. Rev. Lett. 87, 043001 (2001). 
[6] R. B. Vrijen, G. M. Lankhuijzen, and L. D. Noordam, Delayed Electron Emission in the Ionization of Rydberg Atoms with Half-Cycle THz pulses, Phys. Rev. Lett. 79, 617 (1997).

[7] E. Magnuson and T. F. Gallagher, Phase-dependent ionization of Rydberg atoms in static fields, Phys. Rev. A 99, 043415 (2019).

[8] M. L. Zimmerman, J. C. Castro, and D. Kleppner, Diamagnetic Structure of Na Rydberg States, Phys. Rev. Lett. 40, 1083 (1978).

[9] R. J. Elliott, G. Droungas, and J.-P. Connerade, Active cancellation of the motional Stark effect in the diamagnetic spectrum of Ba, J. Phys. B 28, L537 (1995).

[10] K. A. Safinya, J. F. Delpech, F. Gounand, W. Sandner, and T. F. Gallagher, Resonant Rydberg-atom-Rydberg-atom Collisions, Phys. Rev. Lett. 47, 405 (1981).

[11] T. F. Gallagher and P. Pillet, Dipole-dipole interactions of Rydberg atoms, Adv. At. Mol. Opt. Phys. 56, 161 (2008).

[12] F. G. Kellert, K. A. Smith, R. D. Rundel, F. B. Dunning, and R. F. Stebbings, Collisions of xenon $(n f)$ Rydberg atoms with ammonia, J. Chem. Phys. 72, 3179 (1980).

[13] V. Zhelyazkova and S. D. Hogan, Electrically tuned Förster resonances in collisions of $\mathrm{NH}_{3}$ with Rydberg He atoms, Phys. Rev. A 95, 042710 (2017).

[14] V. Zhelyazkova and S. D. Hogan, Probing resonant energy transfer in collisions of ammonia with Rydberg helium atoms by microwave spectroscopy, J. Chem. Phys. 147, 244302 (2017).

[15] F. Jarisch and M. Zeppenfeld, State resolved investigation of Förster resonant energy transfer in collisions between polar molecules and Rydberg atoms, New J. Phys. 20, 113044 (2018).

[16] D. Meschede, H. Walther, and G. Müller, One-Atom Maser, Phys. Rev. Lett. 54, 551 (1985).

[17] S. Gleyzes, S. Kuhr, C. Guerlin, J. Bernu, S. Deléglise, U. Busk Hoff, M. Brune, J. M. Raimond, and S. Haroche, Quantum jumps of light recording the birth and death of a photon in a cavity, Nature (London) 446, 297 (2007).

[18] I. I. Rabi, N. F. Ramsey, and J. Schwinger, Use of rotating coordinates in magnetic resonance problems, Rev. Mod. Phys. 26, 167 (1954).

[19] D. A. Anderson, S. A. Miller, G. Raithel, J. A. Gordon, M. L. Butler, and C. L. Holloway, Optical Measurements of Strong Microwave Fields with Rydberg Atoms in a Vapor Cell, Phys. Rev. Appl. 5, 034003 (2016).

[20] P. Forn-Díaz, J. J. García-Ripoll, B. Peropadre, J.-L. Orgiazzi, M. A. Yurtalan, R. Belyansky, C. M. Wilson, and A. Lupascu, Ultrastrong coupling of a single artificial atom to an electromagnetic continuum in the nonperturbative regime, Nat. Phys. 13, 39 (2017).

[21] J. E. Palmer and S. D. Hogan, Electric Rydberg-Atom Interferometry, Phys. Rev. Lett. 122, 250404 (2019).

[22] C. Deng, F. Shen, S. Ashhab, and A. Lupascu, Dynamics of a two-level system under strong driving: Quantum-gate optimization based on Floquet theory, Phys. Rev. A 94, 032323 (2016).

[23] T. F. Gallagher, Rydberg Atoms (Cambridge University Press, Cambridge, 1994).

[24] H. Fan, S. Kumar, J. Sedlacek, H. Kübler, S. Karimkashi, and J. P. Shaffer, Atom based RF electric field sensing, J. Phys. B 48, 202001 (2015).
[25] C. L. Holloway, M. T. Simons, J. A. Gordon, A. Dienstfrey, D. A. Anderson, and G. Raithel, Electric field metrology for SI traceability: Systematic measurement uncertainties in electromagnetically induced transparency in atomic vapor, J. Appl. Phys. 121, 233106 (2017).

[26] Y. Ni, P. Xu, and J. D. D. Martin, Reduction of the dc-electricfield sensitivity of circular Rydberg states using nonresonant dressing fields, Phys. Rev. A 92, 063418 (2015).

[27] P. Rabl, D. DeMille, J. M. Doyle, M. D. Lukin, R. J. Schoelkopf, and P. Zoller, Hybrid Quantum Processors: Molecular Ensembles as Quantum Memory for Solid State Circuits, Phys. Rev. Lett. 97, 033003 (2006).

[28] S. D. Hogan, J. A. Agner, F. Merkt, T. Thiele, S. Filipp, and A. Wallraff, Driving Rydberg-Rydberg Transitions from a Coplanar Microwave Waveguide, Phys. Rev. Lett. 108, 063004 (2012).

[29] J. D. Pritchard, J. A. Isaacs, M. A. Beck, R. McDermott, and M. Saffman, Hybrid atom-photon quantum gate in a superconducting microwave resonator, Phys. Rev. A 89, 010301(R) (2014).

[30] S. Merz, N. Vanhaecke, W. Jäger, M. Schnell, and G. Meijer, Decelerating molecules with microwave fields, Phys. Rev. A 85, 063411 (2012).

[31] P. Hyafil, J. Mozley, A. Perrin, J. Tailleur, G. Nogues, M. Brune, J. M. Raimond, and S. Haroche, Coherence-Preserving Trap Architecture for Long-Term Control of Giant Ryberg Atoms, Phys. Rev. Lett. 93, 103001 (2004).

[32] S. D. Hogan, Rydberg-Stark deceleration of atoms and molecules, EPJ Tech. Instrum. 3, 2 (2016).

[33] A. Deller and S. D. Hogan, Microwave spectroscopy of the $1 s n p^{3} P_{J}$ fine structure of high Rydberg states in ${ }^{4} \mathrm{He}$, Phys. Rev. A 97, 012505 (2018).

[34] J. H. Shirley, Solution of the Schrödinger equation with a Hamiltonian periodic in time, Phys. Rev. 138, B979 (1965).

[35] P. Pillet, R. Kachru, N. H. Tran, W. W. Smith, and T. F. Gallagher, Radiative Rydberg-atom-Rydberg-atom collisions in the strong-field regime, Phys. Rev. A 36, 1132 (1987).

[36] G. W. F. Drake, High precision theory of atomic helium, Phys. Scr. T83, 83 (1999).

[37] V. Zhelyazkova and S. D. Hogan, Rydberg-Stark states in oscillating electric fields, Mol. Phys. 113, 3979 (2015).

[38] M. L. Zimmerman, M. G. Littman, M. M. Kash, and D. Kleppner, Stark structure of the Rydberg states of alkali-metal atoms, Phys. Rev. A 20, 2251 (1979).

[39] R. N. Zare, Angular Momentum (Wiley, New York, 1988).

[40] T. Halfmann, J. Koensgen, and K. Bergmann, A source for a high-intensity pulsed beam of metastable helium atoms, Meas. Sci. Tech. 11, 1510 (2000).

[41] S. D. Hogan, Y. Houston, and B. Wei, Laser photoexcitation of Rydberg states in helium with $n>400$, J. Phys. B 51, 145002 (2018).

[42] S. H Autler and C. H. Townes, Stark effect in rapidly varying fields, Phys. Rev. 100, 703 (1955).

[43] C. Cohen-Tannoudji, J. Dupont-Roc, and G. Grynberg, Atomphoton Interactions: Basic Processes and Applications (John Wiley \& Sons, Inc., New York, 1998).

[44] F. Bloch and A. Siegert, Magnetic resonance for nonrotating fields, Phys. Rev. 57, 522 (1940).

[45] J. Tuorila, M. Silveri, M. Sillanpää, E. Thuneberg, Y. Makhlin, and P. Hakonen, Stark Effect and Generalized Bloch-Siegert 
Shift in a Strongly Driven Two-Level System, Phys. Rev. Lett. 105, 257003 (2010)

[46] B. Zaks, D. Stehr, T.-A. Truong, P. M. Petroff, S. Hughes, and M. S Sherwin, THz-driven quantum wells: Coulomb interactions and Stark shifts in the ultrastrong coupling regime, New J. Phys. 13, 083009 (2011).

[47] A. A. Morgan, V. Zhelyazkova, and S. D. Hogan, Preparation of circular Rydberg states in helium with $n \geqslant 70$ using a modified version of the crossed-fields method, Phys. Rev. A 98, 043416 (2018).

[48] P. Lancuba and S. D. Hogan, Transmission-line decelerators for atoms in high Rydberg states, Phys. Rev. A 90, 053420 (2014).

[49] P. Lancuba and S. D. Hogan, Electrostatic trapping and in situ detection of Rydberg atoms above chip-based transmission lines, J. Phys. B 49, 074006 (2016). 